\title{
GALACTIC DYNAMOS AND DENSITY WAVE THEORY
}

\section{MESTEL}

Ast ronomy Cent re

University of Sussex

Bright on BN1 $9 \mathrm{QH}$, UK

\author{
K SUBRAMANIAN \\ Tata Inst itute \\ Bombay 400005 \\ India
}

A steady density wave in the stellar background of a disk-like galaxy is supposed to force a spiral shock wave in the interstellar gas. The jump in vorticity across the shock leads to a locally enhanced helicity, and so to an $\alpha$-effect which is steady but azimuth-dependent in the frame rotating with the angular velocity $\Omega$ of the density wave. This is simulated by the adoption of the form $\gamma(r, \varphi)=\gamma_{0}(r)+\tilde{\gamma} \cos (2 \varphi-$ $\kappa r)$ for the local dynamo growth rate arising when the standard kinematic dynamo equation is treated by the thin-disk approximation (Ruzmaikin et al 1988). The global magnetic field is proportional to the function $Q$ satisfying

$$
\frac{\partial Q}{\partial \mathrm{t}}-\gamma \mathrm{Q}-\eta \frac{\partial}{\partial \mathrm{r}}\left[\frac{\partial}{\mathrm{r} \partial \mathrm{r}}(\mathrm{r} \mathrm{Q})\right]+(\omega(\mathrm{r})-\Omega) \frac{\partial \mathrm{Q}}{\partial \varphi}=0
$$

where $\eta$ is the turbulent resistivity (for simplicity assumed uniform) and $\omega=V / r$ is the laminar angular velocity of the gas in the inertial frame. We look for solutions of the form $Q(r, \varphi, t)=\exp (\Gamma t) \Sigma q_{m} \Gamma(r) \exp (\operatorname{im} \phi)$, where $m= \pm 1, \pm 2 \ldots, \Gamma$ is a global eigen-value, and the non-vanishing of $\tilde{\gamma}$ couples all odd or all the even $\mathrm{m}$-values. Anticipating that the strong differential rotation will ensure that in the modes with the largest growth-rate the higher-m parts are weak, the equations are truncated, leaving just a pair in $q_{1}, q_{-1}$ to describe a basically bisymmetric $(m=1)$ mode. Approximate treatment by the WKBJ technique suggests that a corotating growing mode (with $\Gamma$ real and positive) will differ significantly from zero over the range between the points where $\tilde{\gamma} / 2=(\omega-\Omega)^{2}$. Numerical solutions have been found for a set of illustrative parameters with $\tilde{\gamma}<\gamma_{0}, V \simeq 208 \mathrm{~km} \mathrm{~s}^{-1}$, corotation occurring at $6.67 \mathrm{kpc}$, and the turbulence parameters close to those in the M51 mode studied by Ruzmaikin et al which extends over $\simeq 1 \mathrm{kpc}$. Three growing corotating modes were found, the fastest extending for $\sim 3 \mathrm{kpc}$, the other two for over $4 \mathrm{kpc}$. The first two grow 2-3 times faster, the third somewhat slower, than the M51 mode.

Speculation The galactic magnetic field not only facilitates star formation but may also bias the mass spectrum towards more massive stars. One is led to ask if a "grand design" stellar density wave is necessary to explain the spiral arm phenomenon, or whether a self-maintaining dynamo-wave will yield a "bootstrap" picture, with both the energy supply and the generation of helicity coming from the effect of the associated star formation on the interstellar medium.

Reference

Ruzmaikin A.A, Shukurov A.M. \& Sokoloff D.D, (1988) Magnetic Fields of Galaxies, Kluwer Acad. Publ., Dordrecht. 